\title{
Flight Dynamics Operations of the TanDEM-X Formation
}

\author{
Ralph Kahle ${ }^{1}$, Benjamin Schlepp ${ }^{2}$, Saika Aida ${ }^{3}$, Michael Kirschner ${ }^{4}$ and Martin Wermuth ${ }^{5}$ \\ German Aerospace Center (DLR), Oberpfaffenhofen, 82234 Wessling, Germany
}

\begin{abstract}
Since end of 2010 the German TerraSAR-X and TanDEM-X satellites are routinely operated as the first configurable single-pass Synthetic Aperture Radar interferometer in space. The two $1340 \mathrm{~kg}$ satellites fly in a $514 \mathrm{~km}$ sun-synchronous orbit. In order to collect sufficient measurements for the generation of a global digital elevation model and to demonstrate new interferometric SAR techniques and applications, more than three years of formation flying are foreseen with flexible baselines ranging from $150 \mathrm{~m}$ to few kilometers. As a prerequisite for the close formation flight an extensive flight dynamics system was established at DLR/GSOC, which comprises of GPS-based absolute and relative navigation and impulsive orbit and formation control. Daily formation maintenance maneuvers are performed by TanDEM- $X$ to counterbalance natural and artificial disturbances. The paper elaborates on the routine flight dynamics operations and its interactions with mission planning and ground-station network. The navigation and formation control concepts and the achieved control accuracy are briefly outlined. Furthermore, the paper addresses nonroutine operations experienced during formation acquisition, frequent formation reconfiguration, formation maintenance problems and space debris collision avoidance, which is even more challenging than for single-satellite operations. In particular two close approaches of debris are presented, which were experienced in March 2011 and April 2012. Finally, a formation break-up procedure is discussed which could be executed in case of severe onboard failures.
\end{abstract}

\section{Introduction}

$\mathrm{T}$ HE primary goal of the TerraSAR-X mission (TSX, launched on $15^{\text {th }}$ June 2007) is the provision of highresolution Synthetic Aperture Radar (SAR) data to both science and commercial users. On $21^{\text {st }}$ June 2010 an almost identical satellite, TanDEM-X (TDX), was launched in order to form the first configurable SAR interferometer employing formation flying with TSX (see Fig. 1). The main objective of the common TanDEM-X (TerraSAR-X add-on for Digital Elevation Measurement) mission is to generate a global digital elevation model (DEM) with unprecedented accuracy as the basis for a wide range of scientific research as well as for commercial DEM production. ${ }^{1}$ After successful completion of the mono-static and bi-static radar instrument commissioning phases the two satellites started the routine operation as a large single-pass SAR interferometer in December 2010. In order to collect sufficient measurements for a global DEM and to demonstrate new interferometric SAR techniques and applications, more than three years of formation flying are foreseen with flexible baselines ranging from $150 \mathrm{~m}$ to few kilometers.

This first section of the paper gives a general overview on the TSX/TDX mission and the orbit control concepts. The second part is devoted to routine operations and summarizes the achieved control performance within the first two years after TDX launch. Furthermore it elaborates on the constraints imposed by ground-station network and interaction with Mission Planning. Formation-relevant safety measures like the radar instrument exclusion zones and the maneuver collision check are discussed. The third chapter gives a look to non-routine operations, such as formation acquisition, formation reconfigurations or space debris collision avoidance. A spacecraft propulsion problem that occurred during close formation flight is addressed too. Finally, a formation break-up procedure is discussed which could be executed in case of severe onboard failures. Chapter four concludes the paper.

\footnotetext{
${ }^{1}$ TerraSAR-X/TanDEM-X Flight Dynamics Manager, DLR GSOC, Ralph.Kahle@dlr.de.

${ }^{2}$ Flight Dynamics Engineer, DLR GSOC, Benjamin.Schlepp@dlr.de

${ }^{3}$ Flight Dynamics Engineer, DLR GSOC, Saika.Aida@dlr.de

${ }^{4}$ Flight Dynamics Engineer, DLR GSOC, Michael.Kirschner@dlr.de

${ }^{5}$ Flight Dynamics Engineer, DLR GSOC, Martin.Wermuth@dlr.de
} 


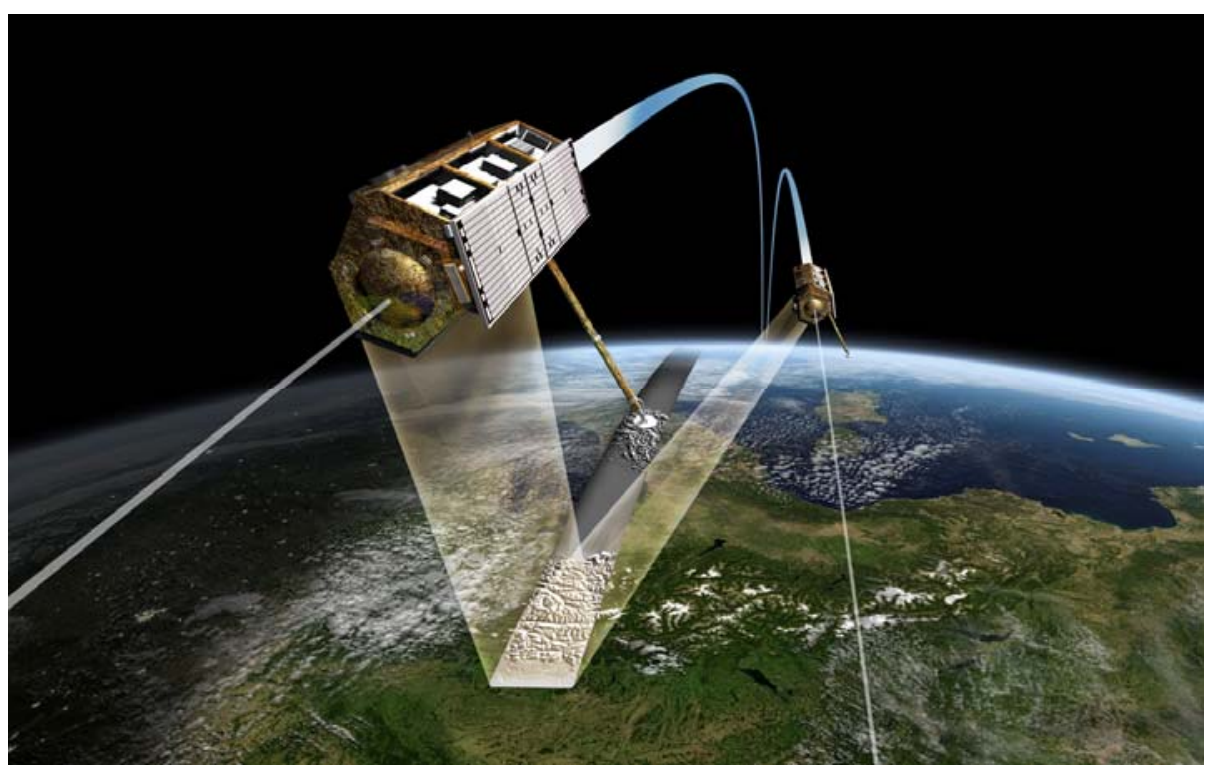

Figure 1. TanDEM-X and TerraSAR-X in close formation flight over Europe.

\section{A. Space-Segment Overview}

The TanDEM-X satellite follows the TerraSAR-X design. Minor modifications are applied, such as an additional cold-gas propulsion system (powered by high-pressure nitrogen gas) to enable fine-tuning of its relative position during formation flying. Furthermore an extra S-band receiver was implemented to receive status and position information sent by TerraSAR-X. This data is utilized within TanDEM-X Autonomous Formation Flying System (TAFF) for purpose of relative navigation and optionally closed loop in-plane formation maintenance. ${ }^{2}$ The TanDEM-X satellite has been designed for a nominal lifetime of five years and has a planned overlap with TerraSAR-X of three years. However, TerraSAR-X holds consumables and resources for up to seven years of operation, potentially allowing for a prolongation of the overlap and the duration of the TanDEM-X formation flying mission. The Flight Dynamics relevant parameters are summarized in Table 1.

Table 1. TerraSAR-X / TanDEM-X satellite characteristics. Flight Dynamics relevant parameters only.

\begin{tabular}{|l|c|c|}
\hline & TerraSAR-X (TSX) & TanDEM-X (TDX) \\
\hline Launch date & $2007-06-15$ & $2010-06-21$ \\
\hline Dimension & \multicolumn{2}{|c|}{ Length: 5 meters, Diameter: 2.4 meters } \\
\hline Mass (2012-05-14) & $1322 \mathrm{~kg}$ & $1325 \mathrm{~kg}$ \\
\hline Propulsion system & $\begin{array}{c}4 \times 1 \mathrm{~N} \text { thrusters in anti-flight } \\
\text { direction }-78 \mathrm{~kg} \text { Hydrazine }\end{array}$ & $\begin{array}{c}4 \times 1 \mathrm{~N} \text { thrusters in anti-flight } \\
\text { direction }-78 \mathrm{~kg} \text { Hydrazine, } \\
2 \times 40 \mathrm{mN} \text { thrusters in each flight and } \\
\text { anti-flight direction - 36 kg Cold-Gas } \\
\text { (Nitrogen) }\end{array}$ \\
\hline GPS receivers & \multicolumn{2}{|c|}{$\begin{array}{c}\text { MosaicGNSS single-frequency (EADS/Astrium), } \\
\text { IGOR dual-frequency (BRE/JPL/GFZ Potsdam) }\end{array}$} \\
\hline Inter-satellite communication & \multicolumn{2}{|c|}{ S-Band receiver } \\
\hline other & \multicolumn{2}{|c|}{$\begin{array}{c}\text { TanDEM-X Autonomous Formation } \\
\text { Flying System (TAFF) }\end{array}$} \\
\hline
\end{tabular}

\section{B. Orbit and Formation Control Concepts}

Since June 2007 the TSX satellite is operated in a sun-synchronous dusk-dawn orbit (97.44 deg inclination, 514 $\mathrm{km}$ altitude, frozen eccentricity) with a 167 orbits or 11-days repeat cycle. The TSX osculating orbit is maintained within a maximum absolute cross-track distance of $250 \mathrm{~m}$ from a target Earth-fixed reference trajectory, which comprises of exactly matching states at beginning and end of the 11-days cycle enabling highly repeatable data-take

2

American Institute of Aeronautics and Astronautics 
conditions. ${ }^{3,4}$ Orbit maneuvers to counteract luni-solar perturbations and to compensate atmospheric drag are performed 3-5 times per year (out-of-plane, up to $30 \mathrm{~cm} / \mathrm{s} \Delta \mathrm{v}$ ) and up to 3 times per week (in-plane, 1 to $5 \mathrm{~cm} / \mathrm{s} \Delta \mathrm{v}$ ) during periods of high solar activity, respectively. ${ }^{5}$ This ground-control strategy is foreseen for the entire TSX lifetime including the period of formation flying with TDX. Thus formation acquisition and maintenance maneuvers are exclusively to be performed by the TDX satellite.

The acquisition of the TanDEM-X DEM requires the coordinated operation of two satellites flying in close formation. The chosen formation geometry implies maximum out-of-plane (cross-track) orbit separation at the equator crossings and maximum radial separation at the poles. This is realized by small ascending node differences and by slightly different eccentricity vectors, respectively, as depicted in Fig. 2. This concept of relative eccentricity / inclination vector separation results in a helix-like relative motion of the satellites along the orbit and provides a maximum level of passive safety in case of vanishing along-track separation. ${ }^{6}$
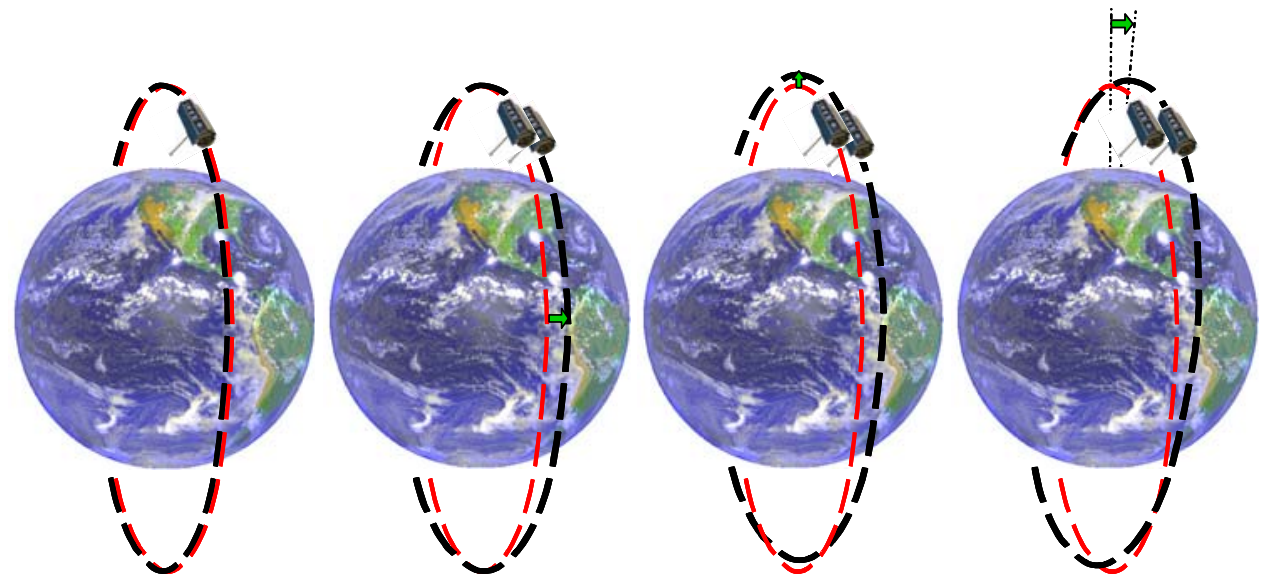

Figure 2. Formation building with relative eccentricity / inclination vector separation. From left to right: (1) identical orbits, (2) horizontal separation at equator crossings by small offset in the ascending node (green arrow indicates RAAN offset), (3) small eccentricity offset causes different heights of perigee / apogee and hence yields radial separation at poles. (4) Optional rotation of the argument of perigee to achieve larger baselines at high latitude regions.

Because of the near-circular low-Earth orbit and the mutual distance on the order of $1 \mathrm{~km}$, linearized equations can be used to describe the TDX-TSX relative motion. The model employed builds upon the well-known ClohessyWiltshire-Hill equations. This provides relative orbital elements as independent parameters and considers secular perturbations on the relative eccentricity and inclination vectors due to the Earth's oblateness (J2). ${ }^{6,7}$

The right ascension of ascending node (RAAN) experiences a drift over the time. When choosing identical inclinations for TSX and TDX, the ascending nodes of their orbits rotate at the same angular velocity yielding stable horizontal baselines as foreseen for the TDX commissioning phase. However, for the DEM acquisition phase the horizontal baseline has to be adjusted frequently. Here, a small inclination offset in the TDX orbit is installed, causing different RAAN drifts to build up larger horizontal baselines over time without the need for expensive RAAN adjustment maneuvers. ${ }^{8}$

The relative eccentricity vector, on the other hand, reflects the secular perigee variation of the individual satellites. It performs a rotation about the origin of the relative eccentricity vector plane with a period of roughly 100 days. The resulting deformation of the relative motion ellipse is illustrated in Fig. 3 (left) over a 24 hours interval. This drift needs to be compensated by suitable in-plane formation keeping maneuvers to maintain a stable configuration. In-plane maneuvers also have an effect on the relative semi-major axis, which results in an alongtrack velocity change. For example, a $300 \mathrm{~m}$ vertical separation demands every day two burns of approx. $0.5 \mathrm{~cm} / \mathrm{s}$ maneuver size each and separated by half a revolution. ${ }^{9}$ These maneuvers are additionally used to adjust the alongtrack separation and to compensate possible differential drag effects. Fig. 3 (right) depicts the uncorrected drift in along-track direction resulting from a $1 \mathrm{~m}$ offset in the semi-major axis of TDX relative to TSX. 

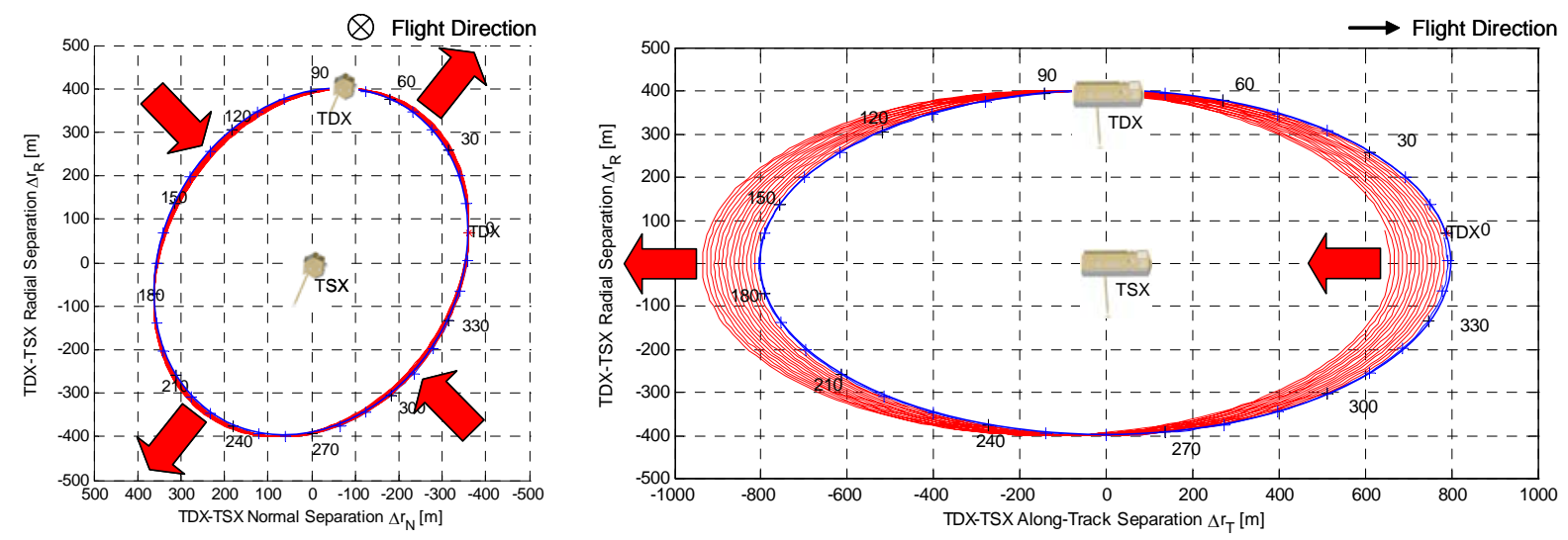

Figure 3. TDX-TSX relative motion in the Hill frame. Once per orbit around Earth, which takes about 95 minutes, TDX moves in a counter-clockwise elliptical path around TSX. Initial (blue) and perturbed evolution within 24 hours or 15 relative orbits (red). Left: Change of Radial / Normal separation induced by Earth's oblateness perturbation on the relative eccentricity vector. Right: Along-track drift when applying a $1 \mathrm{~m} \mathrm{TDX-}$ TSX semi-major axis offset. NOTE: the satellite illustrations are oversized.

\section{Routine Flight Dynamics Operations}

Within the TanDEM-X project GSOC Flight Dynamics (FD) is responsible for

- absolute and relative orbit determination,

- attitude determination and analysis,

- ground station tracking support,

- provision of orbit- and formation-related information for mission planning,

- provision of orbit and attitude products for SAR processing, and

- orbit and formation control.

The complete FD system design and implementation including the development of all software modules was performed by GSOC's space flight technology department.

The focus of this section is on the routine FD operations that are relevant to formation flying. Besides the FD system commissioning phase, these tasks are typically performed by automated ground software and scripts ensuring the compatibility with tight orbit control and product latency requirements. Of course, the FD system is closely monitored by FD engineers and a variety of checks and automated warnings has been implemented to ease problem detection and recovery.

\section{A. Operational Constraints}

Ground-Station Contacts: Initially the TSX telemetry and command (TMTC) network for routine operations only comprised of the Weilheim ground-station offering command and dump capacity typically twice in the morning from approx. 4:30 to 7:00 UTC and twice in the evening from approx. 15:30 to 18:00 UTC. In general, the period inbetween two successive contacts (i.e. 95 minutes) is too short for the entire process from data dumping, over maneuver planning, to command upload. Furthermore, the amount of data that can be dumped within one contact is typically less than 6 hours. Considering the time for gathering all telemetry data necessary to calibrate an executed orbit maneuver and for planning and uploading a new one, the minimum achievable maneuver cycle (i.e. the period between two independently planned maneuvers) is 24 hours for the single Weilheim station scenario. With the use of the Neustrelitz station for TDX, both the coverage and hence the maneuver cycle duration are not affected.

In nominal cases the formation control maneuvers would then take place not earlier than 24 hours after simultaneous execution of absolute TSX/TDX orbit control maneuvers. A $3 \%$ relative execution error of a $5 \mathrm{~cm} / \mathrm{s}$ along-track maneuver changes the relative semi-major axis by almost $3 \mathrm{~m}$ and thus introduces a drift of $24 \mathrm{~m}$ per orbit or $370 \mathrm{~m}$ per day (for comparison see Fig. 3 which depicts a $1 \mathrm{~m}$ semi-major axis offset). Therefore, a 1 day cycle is not sufficient to achieve a $200 \mathrm{~m}$ along-track control accuracy.

Even though the formation control accuracy was the initial driver for a 6 hours TMTC interval, safety concerns that rose during the TanDEM-X development phase became even more stringent. Major concerns are the risk of 
mutual radar illumination and the collision risk after AOCS safe mode drop with possible use of hydrazine thrusters for attitude control (for details refer to Ref. 10). Thus the TMTC routine network was extended by O'Higgins (Antarctica) and Inuvik (Canada) to support midnight contacts and Svalbard to support contacts at noon.

Mission Planning Timeline: The nominal TSX/TDX attitude is not exactly aligned with the orbit-defined rollpitch-yaw coordinates and slightly differs in pitch and yaw depending on the orbit position. In order to maneuver with the hydrazine thrusters the attitude has to be changed disabling the acquisition of SAR data-takes for typically 10 minutes duration. Furthermore the acceleration of the four $1 \mathrm{~N}$ thrusters would disturb focusing within on-ground SAR processing. The instrument timeline is planned twice a day by the Mission Planning System approximately at 10:00 and 22:00 UTC for upload at the evening and morning Weilheim/Neustrelitz contacts, respectively. To consider maneuver outage times the maneuver information has to be available at MPS before the start of the planning run and hence minimum 8 hours before on-board execution.

On the contrary, no instrument interruption is required for the TDX in-plane formation maintenance maneuvers which are performed with the cold-gas thrusters pointing in (anti-)flight direction. Here a cross-coupling of up to 6\% of the in-plane $\Delta \mathrm{v}$ (for maximum 3.7 deg yaw offset) in out-of-plane direction is tolerated. Although the acceleration caused by this maneuver type is too small to negatively affect SAR processing, it could affect the processing of bistatic data-takes acquired by both satellites if the reconstructed baseline experiences changes in its bias or drift. However, the common occurrence is generally avoided by the DEM acquisition strategy foreseeing bi-static datatakes at argument of latitudes of 0 to 90 and 180 to $270 \mathrm{deg}$ while the in-plane formation control maneuvers are typically at 330 to 360 and 150 to $180 \mathrm{deg}$. Thus cold-gas maneuvers for formation maintenance can be planned and commanded on short notice while hydrazine maneuvers are to be prepared on a longer time scale.

\section{B. Navigation}

The fast and accurate orbit determination within the TanDEM-X mission is of paramount importance for the purpose of absolute and relative orbit control, and SAR processing. After every data dump, the TOR-IGOR dualfrequency GPS receiver (or single-frequency MosaicGNSS receiver as backup) telemetry data is pre-processed to extract GPS navigation data for use within Rapid Orbit Determination (ROD) process and raw data for Precise Orbit Determination (POD). The (relative) orbit determination process is dump-triggered and therefore automatically runs 8 to 10 times a day.

The ROD performs a least-squares batch adjustment of the following estimation parameters: epoch state vector (position and velocity), drag coefficient, solar radiation coefficient, extended maneuvers, and measurement biases. As a result of ROD and orbit prediction, two product types are made available to the SAR processing facilities: (a) Predicted orbit product with $700 \mathrm{~m}$ required and $<100 \mathrm{~m}$ achieved accuracy (along-track, 1-sigma, 24h prediction); (b) Quick-look orbit product with $10 \mathrm{~m}$ required and $3 \mathrm{~m}$ achieved accuracy (3D, 1-sigma).

Based on the ROD results, orbit-related products are regularly generated and distributed, e.g. pointing data and two-line elements for S-Band ground station support, input to mission operations sequence of event planning, and CCSDS orbit data messages for laser tracking support.

Within the more precise POD process GPS carrier phase and pseudo-range data are used. Auxiliary data such as the GPS orbit and clock products, Earth orientation parameters, and S/C attitude information are acquired prior to the generation of the precise orbits. The latency of the auxiliary data drives the availability of individual POD product types, i.e.: (c) Rapid orbit product with $2 \mathrm{~m}$ required and $0.1 \mathrm{~m}$ achieved accuracy (3D, 1-sigma); (d) Science orbit product with $20 \mathrm{~cm}$ required and $5 \mathrm{~cm}$ achieved accuracy (3D, 1 -sigma). ${ }^{11}$

\section{Relative Navigation}

The formation monitoring process is based on the synchronous TSX/TDX rapid orbit determination from navigation solution data. The processing of equal data arcs with same environment model parameters cancels common errors which are mainly related to atmospheric drag modeling. Typically, the relative navigation as used in the formation control process has a cross-track (2D) accuracy $<0.5 \mathrm{~m}$ (RMS), which is sufficient for the purpose of formation monitoring and control with specified $20 \mathrm{~m}$ control accuracy in cross-track. In along-track direction the navigation accuracy is on the $1 \mathrm{~m}$ level where $200 \mathrm{~m}$ control accuracy is required. ${ }^{8}$

In order to process the DEM data-takes with highest accuracy, the TDX-TSX relative position has to be known with an accuracy of $1 \mathrm{~mm}(1 \mathrm{D}, \mathrm{RMS})$. The vector between the SAR antenna reference points of the two satellites, the so-called baseline, is determined with the FRNS software (Filter for Relative Navigation of Spacecraft) developed at DLR/GSOC in cooperation with the TU Delft. It is based on an extended Kalman filter/smoother process. The underlying concept of the FRNS software is to achieve a higher accuracy for the relative orbit between two spacecraft by making use of differenced GPS observations, rather than by simply differencing two independent precise orbit determination results. The use of single-differenced code and carrier phase observations rigorously 
eliminates GPS clock offset uncertainties and largely reduces the impact of GPS satellite orbit and phase pattern errors. Double differences are used for the integer ambiguity resolution of the carrier phase observations and common error cancellation. One of the major error sources is the ionospheric delay, which cancels out to a large degree due to the short distance between the two spacecraft. Current analysis confirms standard deviation in the lineof-sight vector below $1.5 \mathrm{~mm} .{ }^{12}$

\section{Formation Maintenance}

The starting point of TDX-TSX formation control operation is the TanDEM-X acquisition plan, which contains the timeline of formation geometries and corresponding planned bi-static SAR acquisitions. The target formation parameters are provided to FD, where they are converted into relative orbital elements before applying them within the formation control process.

For the purpose of SAR cross-track interferometry, TDX must be kept in close proximity of TSX. In order to meet the tight relative control requirements (i.e. $20 \mathrm{~m}$ perpendicular and $200 \mathrm{~m}$ parallel to flight-direction) TDX has to (a) compensate the natural deviation of the relative eccentricity vector, (b) counteract the natural drift of the inclination vector, (c) control the along-track separation which is perturbed by relative drag and in-plane maneuver execution errors, and (d) replicate the TSX orbit keeping maneuvers. The out-of-plane relative orbit maintenance and the larger absolute orbit maintenance maneuvers $(b, d)$ are performed with four 1 Newton hydrazine thrusters. For the in-plane formation control (a, c) exclusively two pairs of $40 \mathrm{mN}$ cold-gas thrusters are used.

Shortly after acquiring the narrow formation in October 2010 the ground formation control process became fully automated. Daily TDX in-plane maneuver pairs with typically 3 to $10 \mathrm{~mm} / \mathrm{s}$ maneuver size are performed to compensate the natural eccentricity vector drift and to adjust the along-track separation (a, c). Out-of-plane control is used to adjust the horizontal separation, by either changing the relative inclination and therefore the drift of the relative RAAN or directly by adjusting the RAAN for larger reconfigurations. Two out-of-plane maneuvers are separated by a minimum time interval of 11 days. The achieved relative control accuracy is nominally $<5 \mathrm{~m}$ (RMS) in cross-track direction (i.e. 2D) and can be up to $10 \mathrm{~m}$ (RMS) during phases of large horizontal separation drifts as

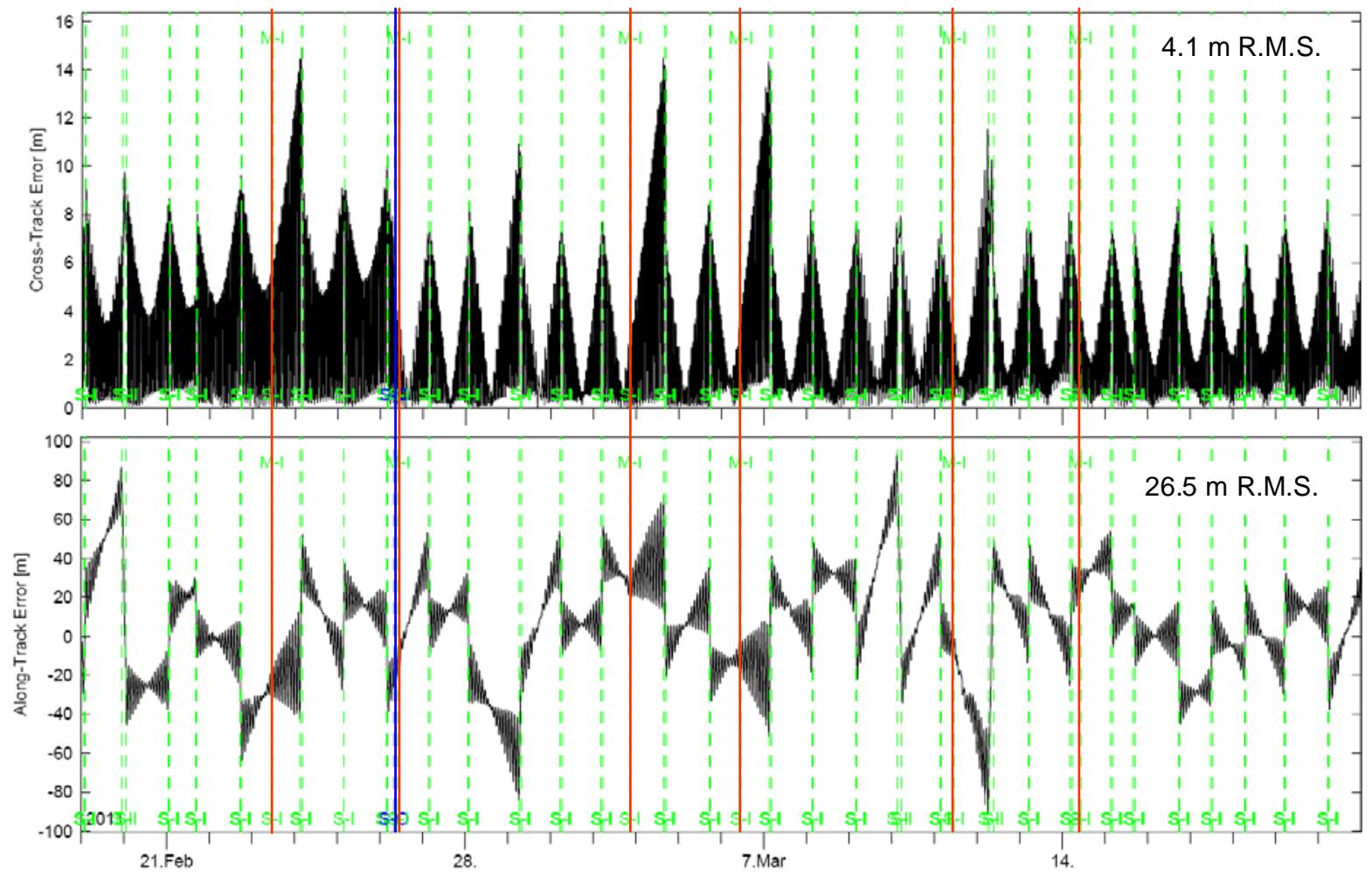

Figure 4. Formation control performance (Feb. 19 to Mar. 21, 2011). Top: the combined radial and normal error (i.e. cross-track error, 2D) is $4.1 \mathrm{~m}$ R.M.S. (requirement: $28.3 \mathrm{~m}$ ). Bottom: the along-track error amounts to $26.5 \mathrm{~m}$ R.M.S. (requirement: $200 \mathrm{~m}$ ). The vertical lines indicate common TSX/TDX maneuvers (red) and TDX formation maintenance maneuvers (green: in-plane, blue: out-of-plane). 
performed in the beginning of the DEM acquisition phase. The along-track control accuracy is better than $30 \mathrm{~m}$ (RMS). The control requirements of 28/200 m (RMS) in cross-track/along-track direction are clearly overachieved. Fig. 4 depicts the stable and precise control of the TDX-TSX cross-track and along-track separation over a 30-days period.

So far, more than 1,000 formation control maneuvers were performed by TDX within 1.5 years of close formation flying with TSX. The biggest challenge as compared to previous experimental formation flying missions is the operational character demanding for continuously safe and robust operation with high control accuracy for at least three years. The frequent maneuver planning process has to consider not only the ground-station network (implying the availability of command upload or navigation data availability) and the interaction with radar instrument operation. Also unforeseen events, e.g. maneuver failures or satellite safe mode drops, must not imply any danger of collision. A maneuver check has been implemented to analyze how the formation changes in consequence of all possible maneuver failure scenarios. For example, the planning of one common TSX/TDX maneuver and two additional TDX formation maintenance maneuvers yields 16 maneuver failure scenarios. If any of these scenarios implies a close approach (e.g. $150 \mathrm{~m}$ in the plane perpendicular to flight-direction) the planned maneuvers are not released for commanding and the automated process stops and requires manual interaction by the FD on-call engineer instead. This is regularly the case for TSX out-of-plane maneuvers which can be up to $30 \mathrm{~cm} / \mathrm{s}$ large, in the worst case resulting in a horizontal separation change of $270 \mathrm{~m}$.

\section{E. Radar Transmission Exclusion Zones}

If one satellite is located in the area illuminated by the SAR antenna main lobe of the other satellite, damage of electronic units could result on the illuminated satellite. Therefore exclusion zones (EZ) have to be defined along the orbit for each satellite, in which the transmission by the SAR antenna is disabled.

As the control accuracy of the relative alongtrack distance between the satellites is relatively low, the EZ definition is neglecting the inherent along-track variations and the EZ location is defined only depending on the elevation angle in the plane perpendicular to flight-direction (Fig. 5). Exclusion zones are determined for both satellites and for both looking directions. For each case the corresponding envelope antenna pattern is used and specific security margins are applied.

Two EZ products are generated by FD: (a) Transmit Exclusion Windows (TEW) for on-board timeline generation within Mission Planning System and, (b) Transmit Exclusion Ephemeris (TEE) for an additional on-ground check within the Monitoring and Control System (MCS).

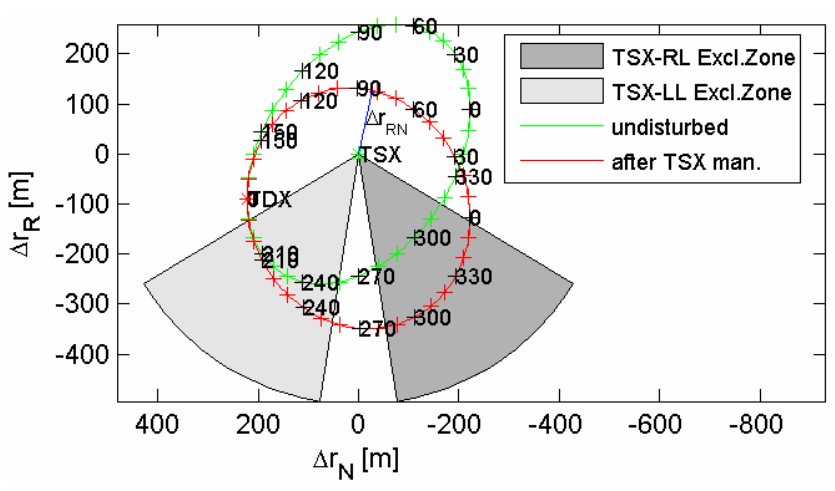

Figure 5. Illustration of radar transmission exclusion zones. TDX-TSX undisturbed motion (green curve) in the plane perpendicular to flight-direction and TSX right-and left-looking (RL, LL) exclusion zones (grey). Ticks indicate argument of latitude (AOL) positions. At $180 \mathrm{deg}$ AOL a maneuver is executed on TSX but fails on TDX. The perturbed relative motion and corresponding exclusion zone AOLs are shown in red. As a consequence TDX exits the TSX right-looking EZ at AOL of 358 deg instead of nominally $347 \mathrm{deg}$.

In order to avoid mutual illumination due to unforeseen formation geometry after non-execution of orbit maneuvers, FD computes extended transmit exclusion products for a period starting after any hydrazine maneuver and lasting for a pre-defined interval. For example, if one planned TSX maneuver has to be replicated by TDX, four cases are considered in the EZ product generation. This is necessary because e.g. the non-execution of a $1 \mathrm{~cm} / \mathrm{s}$ maneuver may cause the illuminated satellite to stay 100 seconds longer in the exclusion zone compared to the nominally computed EZ product (Fig. 5). After reception of TEWs MPS checks in the first place whether or not requested data-takes fall into transmit exclusion windows. For data-take requests outside TEWs command sets are sent to the MCS. To achieve redundancy, a second "Late Check" based on the TEE Product is performed in MCS before the actual data-take commands are up-linked to the satellites.

\section{Non-Routine Flight Dynamics Operations}

This section addresses non-routine operations that have been experienced during formation acquisition, regular formation reconfiguration and space debris collision avoidance, which can be even more challenging compared to 
single-satellite operations. Finally, an emergency procedure is discussed, which aims on maneuvering one satellite into a safe distance from the other one.

\section{A. Formation Acquisition}

After the TDX launch on $21^{\text {st }}$ June 2010 from Baikonur TDX was $15,700 \mathrm{~km}$ behind of TSX. Shortly after the commissioning of main AOCS sensors and actuators, a first orbit control maneuver could be performed within 1.5 days after launch to adjust the along-track drift rate towards TSX to about $630 \mathrm{~km}$ per day. The along-track drift was step-wise reduced and stopped in the period 12-18 July 2010 and the formation geometry foreseen for mono-static radar instrument commissioning was successfully acquired. The total TDX velocity increment contained within 23 in-plane and out-of-plane acquisition maneuvers performed in the period from June 22 to July 19 was only $6.5 \mathrm{~m} / \mathrm{s}$ out of a maximum budget of $18.5 \mathrm{~m} / \mathrm{s}$ (for details cf. Ref. 13).

\section{B. Formation Reconfiguration}

The helix-like formation implies a variation of the effective instrument baseline as a function of argument of latitude (or orbit position). By proper setting of the radar instrument looking angle, a stable baseline (i.e. minimum cross-track distance between TSX and TDX instrument line-of-sight) can be achieved over short latitude region. Southern and northern latitudes are mapped with the same formation by using descending and ascending orbits, respectively. Since the global DEM generation requires the multiple global acquisition with nearly constant baselines, different formation geometries are required depending on the latitude region.

To adjust the baselines, formation reconfiguration maneuvers can be performed by TDX employing hydrazine propulsion. In contrast to the automated routine formation maintenance, the larger reconfiguration maneuvers are manually planned by the FD engineer. The changes in the TDX-TSX formation geometry realized within the year 2011 are summarized in Fig. 6.

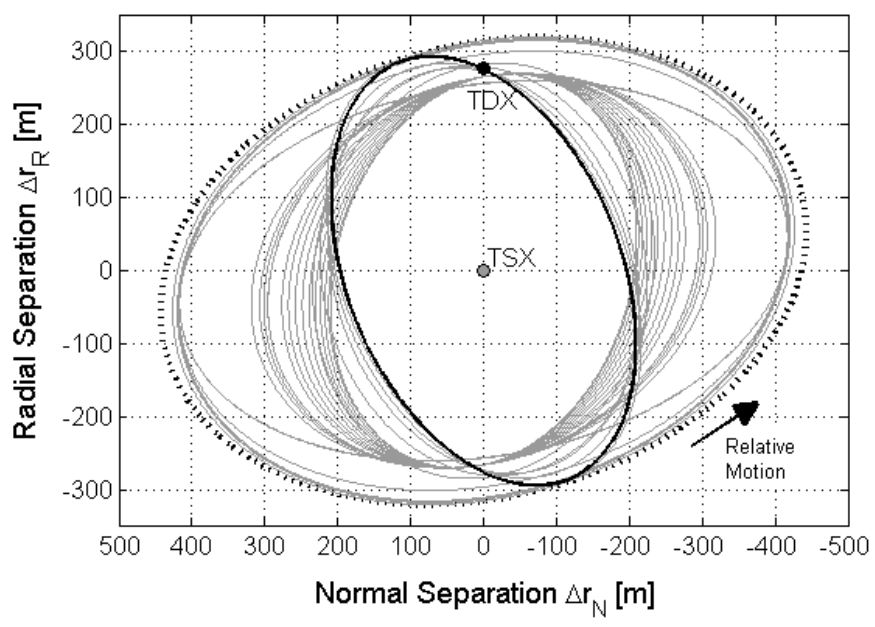

Figure 6. Evolution of TDX-TSX relative motion during the period from Dec. 2010 (dotted ellipse) to Dec. 2011 (bold black ellipse). The relative motion is shown in the plane perpendicular to flight direction. The filled black circle indicates the TDX relative position at 90 deg argument of latitude (north polar crossing).

Because of the tight DEM acquisition schedule, usually only a short period of about 4.5 hours (or 3 orbital periods) is reserved for performing the in-plane and out-of-plane maneuvers for formation reconfiguration. The careful planning of reconfiguration operations typically involves engineers from the disciplines of FD, AOCS, radar instrument, mission planning and mission operation coordination. Together they work out a sequence of events covering ground and space segment activities, e.g. the temporary increase of exclusion zone margins or the upload of attitude steering tables.

\section{Problems during Formation Maintenance}

The usually stable and precise formation control was temporarily degraded in the period from September 17 to 19, 2011. Especially the TDX-TSX along-track separation was affected, which is highly sensitive to small differences in the semi-major axes of TSX and TDX (cf. discussion in Fig. 3). The underlying problem was most likely a not entirely closed TDX cold-gas thruster oriented in anti-flight direction. For details on anomaly detection, recovery and root cause analysis see Ref. 14 .

Fig. 7 depicts the evolution of the TDX-TSX semi-major axis (SMA) difference during the anomalous period. Because of the almost identical satellite masses (approx. 1\% mass difference, cf. Tab. 1) and equal surface area, the satellites only experience a very small relative SMA decay in the order of $10 \mathrm{~cm}$ per day. While the little leakage experienced with TDX would possibly be not detectable by means of single-satellite orbit determination (the uncertainty in drag modeling has a larger impact at $514 \mathrm{~km}$ altitude orbit), the effect can clearly be seen in the 
relative navigation. The blue box in Fig. 7 locates the period of leakage with the resulting $2 \mathrm{~m}$ per day relative SMA decay.

The first maneuvers with back-up cold-gas thruster were performed in the evening of Sept. 19. After the branch switch the remaining gas within the leaking branch depleted and the relative motion perturbation stopped. A sufficient number of maneuvers with the new branch were performed by Sept. 21 allowing for thruster performance characterization and maneuver planning adjustment. Thereafter, the formation control performance was back to nominal. In Fig. 7 (bottom) the along-track formation control accuracy is depicted for a 10-days period clearly showing the significant impact of the small leakage (blue box) and later of the un-calibrated back-up thrusters on the control performance.

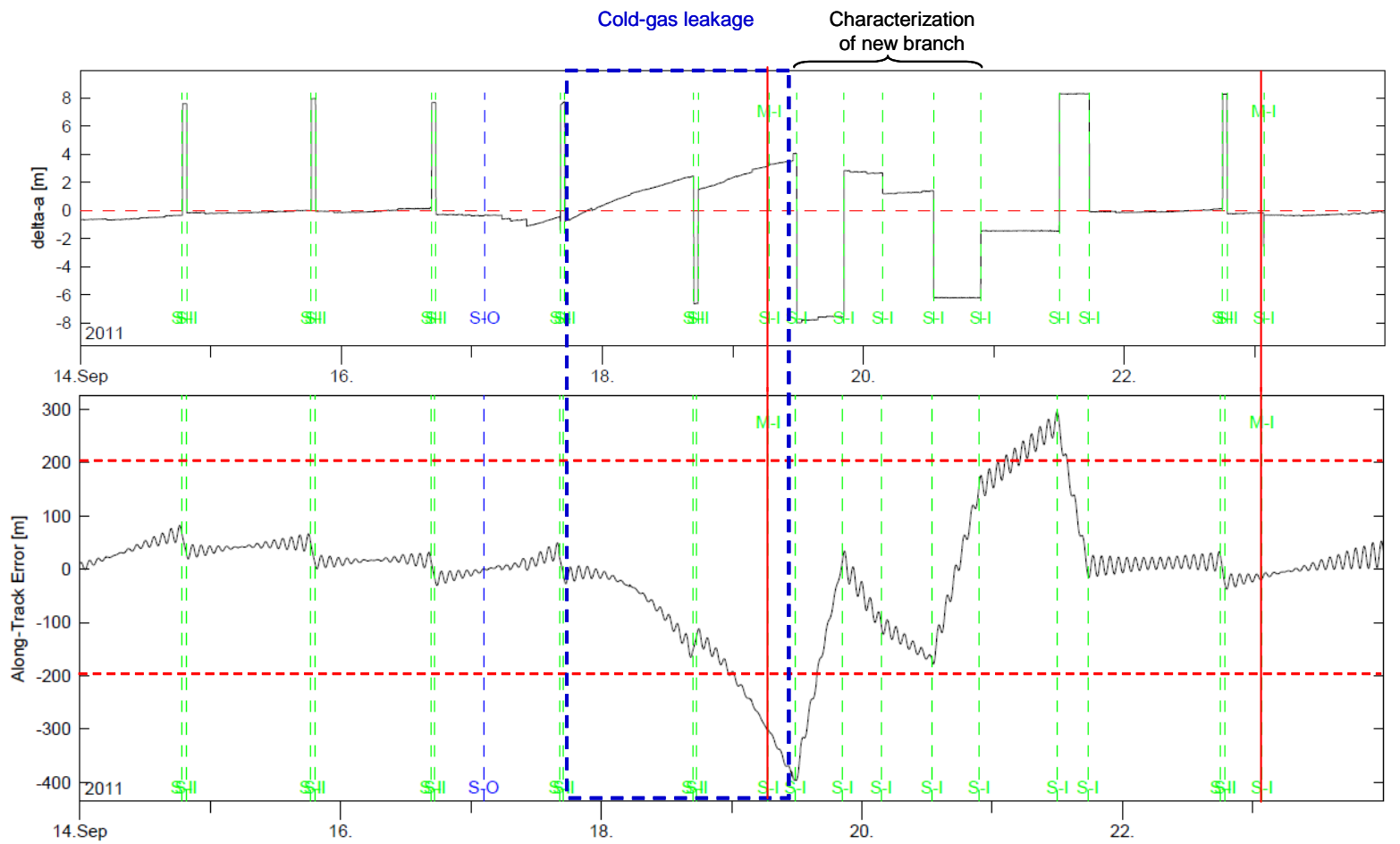

Figure 7. TDX-TSX semi-major axis difference (top) and along-track formation control accuracy (bottom). Period from Sept. 14 to 24, 2011. Violations of the $+/-200 \mathrm{~m}$ along-track control limit (red horizontal lines) occurred as a consequence of a leaking TDX cold-gas thruster in Sept. 17-19 (blue box) which lead to small but permanent lift of the TDX orbit. After branch-switch and thruster performance characterization the nominal control performance was achieved on the evening of Sept. 21. Vertical lines indicate common TSX/TDX maneuvers (red) and TDX formation maintenance maneuvers (green: in-plane, blue: out-of-plane).

\section{Space Debris Collision Avoidance}

GSOC FD performs a semi-daily collision risk assessment for all GSOC-operated satellites (for details cf. Ref. 15). Depending on approach geometry and risk estimate a ground radar tracking campaign can be made to re-asses the risk. Due to the high safety and orbit control requirements of TSX and TDX a dedicated maneuver strategy has to be considered to handle close approaches with space debris.

If the risk applies only to TSX the following collision avoidance scenarios exist:

1) The execution time and size of a regular TSX maneuver is changed so that the maneuver takes place before (or after) the event. TDX replicates the maneuver as usual.

2) TSX performs two maneuvers: collision avoidance and re-acquisition of reference orbit, and

2.1) TDX replicates the maneuvers, which can be fuel-expensive, or

2.2) TDX remains passive and the formation has to be re-acquired by TDX afterwards, which can be timeconsuming.

Case 1) might only be an option when collision event and planned routine maneuver epoch are within 24 hours. Clearly, in case 2) a trade-off has to be made when deciding about the avoidance strategy. 
If solely TDX is affected, TSX remains passive and TDX has to perform maneuvers for collision avoidance and formation re-acquisition.

Due to such a complex operation, the collision avoidance maneuver planning process has to be performed manually by FD engineers. Usually the avoidance maneuver commands are prepared quite late (i.e. $<12$ hours before the execution epoch) compared to routine orbit maneuvers. Then, in order to minimize the impact on the radar missions the maneuver epochs are often to be harmonized with the finalized SAR acquisition timeline. In the following two recent critical approaches are presented, which lead to the execution of a collision avoidance maneuver after a careful risk assessment.

The close approach of TDX to CZ-4 Debris (approx. $15 \mathrm{~cm}$ size estimated from radar cross section) at 2011/03/25 15:08:11 UTC was the first critical close approach since the close formation of TSX and TDX was acquired in October 2010. Only 12 hours before the time of closest approach (TCA), a warning from the Joint Space Operation Center (JSpOC) was received announcing total/radial distances of $84 / 83 \mathrm{~m}$ for TDX and 245/165 $\mathrm{m}$ for TSX. The risk was re-assessed by FD making use of precise orbital elements of the satellites (GSOC POD, cf. II. B.) and orbit data of the debris provided by JSpOC. The updated results confirmed a critical proximity of CZ-4 debris, passing through the close formation as depicted in Fig. 8. The TDX-TSX relative motion is given as the blue curve plotted in the plane perpendicular to the flight direction. The pink error bars show the $20 \mathrm{~m}(1-\sigma)$ radial and normal formation control requirement of TDX. The uncertainty of the debris is displayed by the red band (3- $\sigma$ ellipse). Compared to the estimated orbit uncertainties (1- $\sigma$ radial of $2 \mathrm{~m}$ for TDX, and $20 \mathrm{~m}$ for the debris), the predicted TDX position at TCA was found to be barely outside the 3- $\sigma$ region. Because of the very close approach (intersection between pink bars and red band) and the unavailability of ground radar measurements for refined debris orbit determination, it was decided to perform a collision avoidance maneuver with TDX solely. A small (i.e. $1 \mathrm{~cm} / \mathrm{s}$ ) tangential maneuver was applied half orbital period before TCA in order to increase the CZ-4 / TDX radial miss-distance by $40 \mathrm{~m}$, bringing TDX to $50 \%$ outside the 3- $\sigma$ uncertainty region from the orange star to the green star relative orbit. Two in-plane maneuvers were performed in total; one for the collision avoidance and the other one for the formation re-acquisition half an orbit after TCA. Because of the avoidance maneuvers, TDX was slightly outside the control band (upper blue curve). The maximum cross-track error was only $37 \mathrm{~m}$ with regard to the target formation parameters and therefore it can be concluded that the SAR instrument operation was not affected.
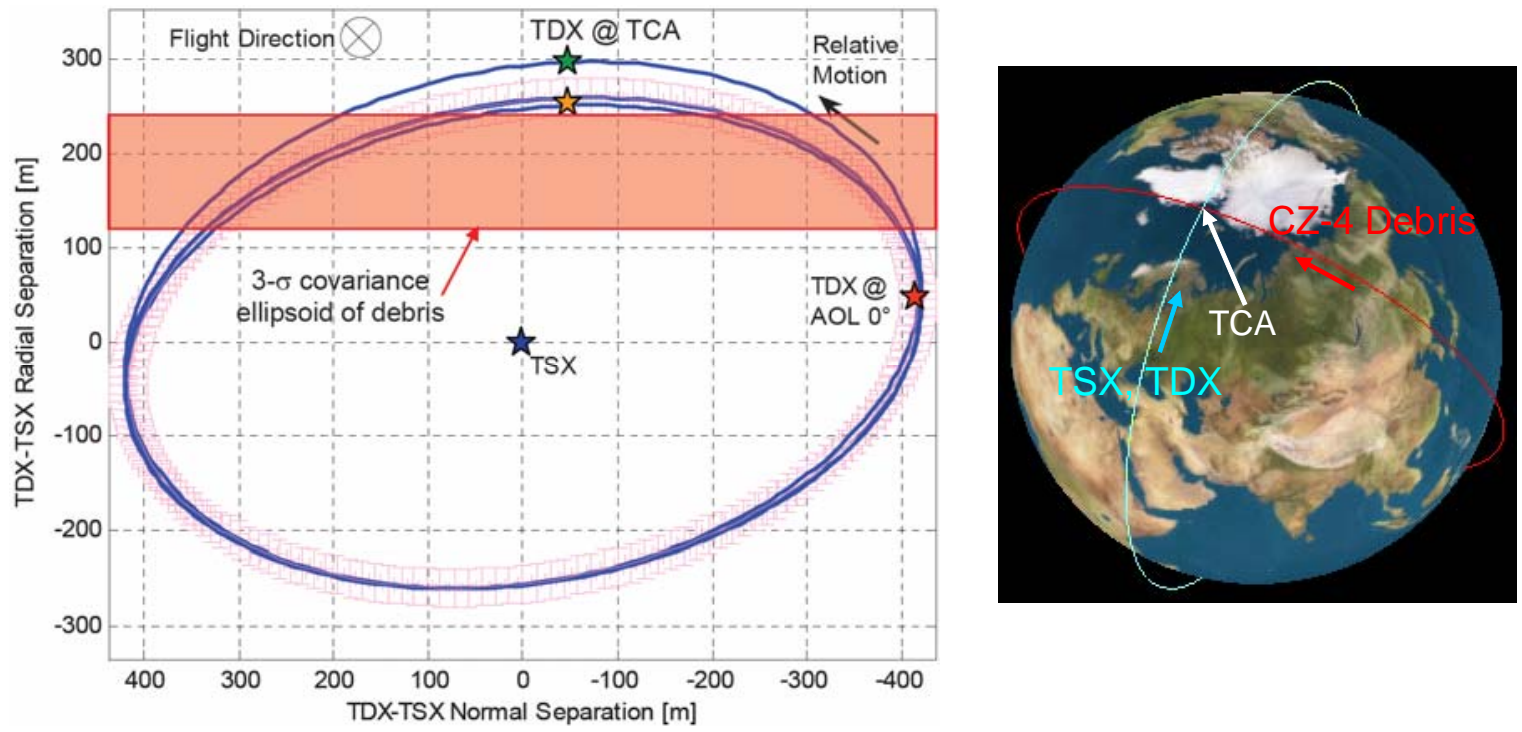

Figure 8. TDX-TSX / CZ-4 relative motion (left) and close approach geometry (right) at TCA (2011/03/25 15:08:11 UTC). Left: CZ-4 debris location is depicted as 3- $\sigma$ covariance ellipsoid (red area). TDX position at TCA after avoidance maneuver execution is shown as green star. Without maneuvering TDX (orange star) would have been very close to the debris uncertainty ellipse. Because of the fact that TSX and debris planes were nearly perpendicular to each other (right), the debris along-track uncertainty directly maps into uncertainty in normal direction within TSX Hill frame (left). Therefore the debris uncertainty appears more like a band than an ellipse.

The given CZ-4 example illustrates the avoidance scenario where solely TDX is affected and maneuvered. Finally, the case 1) will be discussed with an example where TSX was approached by Okean 3 debris with approx. 
$20 \mathrm{~cm}$ size. The time of closest approach (TCA) was 2012/04/21 00:13:04 UTC. One day before TCA, a JSpOC warning for TSX was received with the minimum / radial distance of $676 / 78 \mathrm{~m}$. The re-assessment by GSOC showed a minimum distance / radial distance of only 66 / $65 \mathrm{~m}$ for TSX, and 515 / $515 \mathrm{~m}$ for TDX. Although the TSX radial miss-distance of $65 \mathrm{~m}$ was slightly outside the 3 -sigma threshold, an avoidance maneuver was performed to increase the safety by combining with a planned TSX orbit maintenance maneuver. The regular TSX maneuver was re-planned to be executed one day ahead of schedule to increase the radial distance by $56 \mathrm{~m}$ at TCA. The formation geometry was not affected because TDX replicated this maneuver.

\section{E. Formation Breakup Procedure}

Finally, this section discusses a scenario that fortunately has not been experienced, yet. In case of an onboard failure that could lead to an un-controlled change of the relative motion (e.g. by use of thruster-based attitude safe mode) the ground control would immediately break-up the formation by means of orbit maneuvering of the healthy satellite. To allow for short reaction times, maneuver scenarios are pre-defined by FD, which are dependent on current formation geometry.

Fig. 9 illustrates a scenario where TSX becomes unhealthy. At the first possible TMTC contact after problem detection and decision taking, the TDX satellite would then be commanded to perform an orbit-lowering maneuver at subsequent north-polar crossing (i.e. at argument of latitude of $90 \mathrm{deg}$ ). The relative motion ellipse would then change from the blue towards the red ellipse. The maneuver size is typically $2.8 \mathrm{~cm} / \mathrm{s} \mathrm{implying} \mathrm{a} \mathrm{semi-major} \mathrm{axis}$ change by $50 \mathrm{~m}$ and initiating an along-track drift of approx. $450 \mathrm{~m}$ per orbital period or $7.5 \mathrm{~km}$ per day. This drift would be stopped after 1 day at $7.5 \mathrm{~km}$ safe separation from TSX. The two in-plane maneuvers additionally build-up a radial separation of $100 \mathrm{~m}$ too. After successful recovery of the affected satellite the formation could be reacquired with a similar maneuver scenario within one day only.
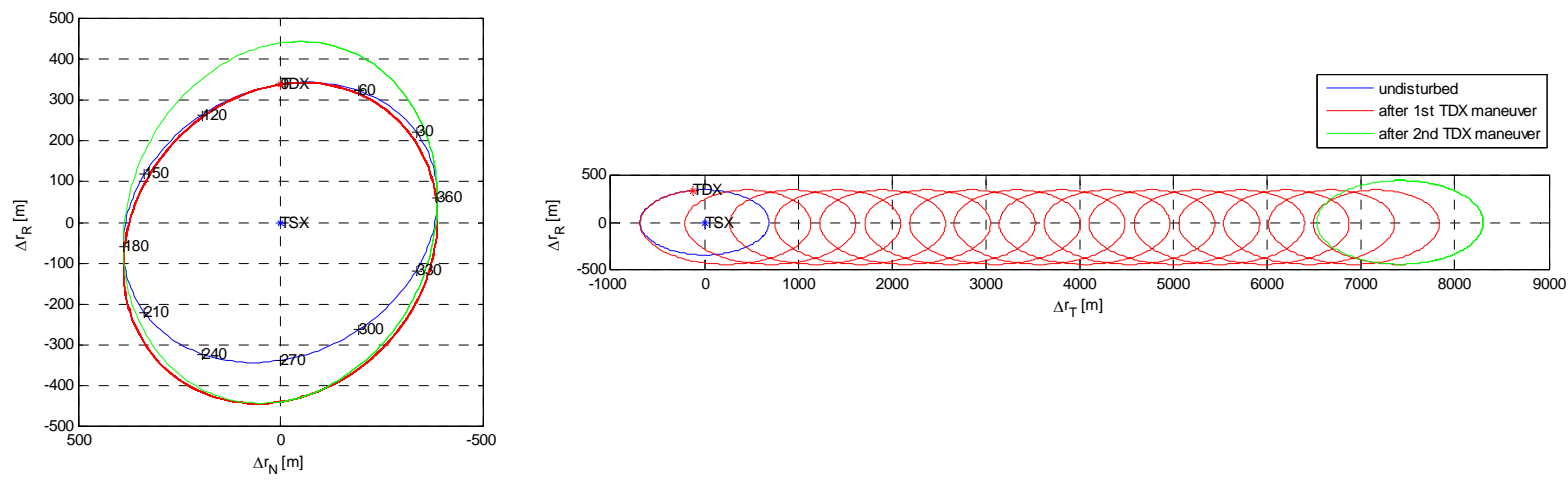

Figure 9. Illustration of formation break-up scenario. TDX performs an orbit lowering maneuver at north polar crossing (transition from blue to red relative motion ellipse). The initialized along-track drift is depicted right. After 1 day a second maneuver is performed to stop the drift at $7.5 \mathrm{~km}$ mean along-track distance from TSX (green ellipse).

\section{Conclusion}

Within the first two years after TDX launch remarkable in-flight results could be achieved comprising the timely and fuel-efficient acquisition of the formation with TSX in July 2010, the safe and precise ground formation control, and the reliable provision of FD products for mission planning and SAR processing. Daily formation maintenance maneuvers are performed by TDX to counterbalance natural and artificial disturbances. The achieved relative control accuracy is nominally $5 \mathrm{~m}$ (RMS) in cross-track direction and $30 \mathrm{~m}$ (RMS) in along-track direction. The control requirements of 28 / $200 \mathrm{~m}$ in cross-track / along-track direction are clearly overachieved. So far, only a minor problem within the formation maintenance process occurred as consequence of a leaking cold-gas thruster in September 2011. The anomaly could easily be identified and after direct recovery the formation control accuracy was quickly back to nominal. The concept developed for debris collision avoidance of formation flying satellites has successfully been applied to TSX and TDX. Operational results in 2009-2011 show that typically two close approaches per year lead to collision avoidance maneuvers, which are planned with minimal impact on scientific and commercial radar acquisition. 
Key technologies like close formation flying and precise baseline determination have successfully been implemented for the TanDEM-X mission. Sophisticated safety mechanisms allow for safe operation at typical distances between $150 \mathrm{~m}$ and few $\mathrm{km}$. The two twin satellites and the ground segment perform remarkably well paving the way for the acquisition and processing of a global digital elevation model within three years of TDXTSX formation flying.

\section{Acknowledgments}

The TanDEM-X project is partly funded by the German Federal Ministry for Economics and Technology (Förderkennzeichen 50 EE 0601) and is realized in a public-private partnership (PPP) between German Aerospace Center (DLR) and Astrium GmbH.

\section{References}

${ }^{1}$ Krieger, G., Moreira, A., Fiedler H., Hajnsek I., Werner M., Younis, M., Zink, M., "TanDEM-X: A satellite formation for high-resolution SAR interferometry", IEEE Transaction on Geoscience and Remote Sensing, Vol. 45, No. 11, Feb. 2010, pp. $3317-3341$

${ }^{2}$ Ardaens, J.S., D'Amico, S. and Fischer, D., "Early Flight Results from the TanDEM-X Autonomous Formation Flying System", Proceedings of the 4th International Conference on Spacecraft Formation Flying Missions \& Technologies, St-Hubert, Quebec, Canada, 2011.

${ }^{3}$ Arbinger, C., D’Amico, S., and Eineder, M., "Precise ground-in-the-loop orbit control for low earth observation satellites", Proceedings of the 18th International Symposium on Spaceflight Dynamics, Munich, Germany, 2004.

${ }^{4}$ D'Amico, S., Arbinger, C., Kirschner, M., Campagnola, S., "Generation of an optimum target trajectory for the TerraSAR X repeat observation satellite", Proceedings of the 18th International Symposium on Spaceflight Dynamics, Munich, Germany, 2004.

${ }^{5}$ Kahle, R., Kazeminejad, B., Kirschner, M., Yoon, Y., Kiehling, R., D’Amico, S., "First In-orbit Experience of TerraSAR-X Flight Dynamics Operations", Proceedings of the 20th International Symposium on Space Flight Dynamics, Annapolis, USA, 2007.

${ }^{6}$ D'Amico, S., and Montenbruck, O., "Proximity operations of formation flying spacecraft using an eccentricity/inclination vector separation", Journal of Guidance, Control, and Dynamics, Vol. 29, 2006, pp. 554-563.

${ }^{7}$ Clohessy, W. H., and Wiltshire, R.S., "Terminal guidance system for satellite rendezvous", Journal of Aerospace Science , Vol. 270, 1960, pp. 653-658.

${ }^{8}$ Kahle, R., Schlepp, B., and Kirschner, M., "TerraSAR X/TanDEM X formation control - first results from commissioning and routine operations", Proceedings of the 22nd International Symposium on Spaceflight Dynamics, Sao Jose dos Campos, Brazil, 2011.

${ }^{9}$ Montenbruck, O., Kahle, R., D'Amico, S., Ardaens, J.-S., "Navigation and Control of the TanDEM-X Formation", Journal of the Astronautical Sciences, Vol. 56, No. 3, 2008, pp. 341-357.

${ }^{10}$ Hofmann, H., and Kahle, R., "The TanDEM-X Mission Operations Segment: Close formation flight: Preparation and First Experiences", AIAA-2010-2195, Proceedings of SpaceOps 2010 Conference, Huntsville, Alabama, USA, 2010.

${ }^{11}$ Wermuth, M., Hauschild, A., Montenbruck, O., Jäggi, A., "TerraSAR-X Rapid and Precise Orbit Determination", Proceedings of the 21st International Symposium on Spaceflight Dynamics, Toulouse, France, 2009.

${ }^{12}$ Wermuth, M., Montenbruck, O., and Wendleder, A., "Relative Navigation for the TanDEM-X Mission and Evaluation with DEM Calibration Results", Proceedings of the 22nd International Symposium on Spaceflight Dynamics, Sao Jose dos Campos, Brazil, 2011.

${ }^{13}$ Kahle, R., Schlepp, B., Meissner, F., Kirschner, M., Kiehling, R., "TerraSAR-X/TanDEM-X Formation Acquisition Analysis and Flight Results", Proceedings of the 21st AAS/AIAA Space Flight Mechanics Meeting, New Orleans, Louisiana, 2011.

${ }^{14}$ Schulze, D., Herman, J., and Löw, S., "Formation Flight in Low-Earth-Orbit at 150 m: AOCS In-Orbit Experience", SpaceOps 2012 Conference, Stockholm, Sweden, 2012.

${ }^{15}$ Aida, S., Kirschner, M., and Kiehling, R., "Collision Avoidance Operations for LEO Satellites Controlled by GSOC", AIAA 2010-2298, Proceedings of SpaceOps 2010 Conference, Huntsville, Alabama, USA, 2010. 